\title{
OMAPL138-based Systematic Study on Portable EEG Detection System
}

\author{
Ze-hua Liu \\ Tianjin Information Sensing and Intelligent Control Laboratory, Tianjin University of Technology and Education No.1310,Dagu South Road,
} Hexi District, Tianjin 300222, China

\begin{abstract}
OMAPL138-based portable EEG detection system is designed in this paper. This system transplants algorithm to OMAPL138 processor to realize preprocessing, storage and dynamic display of data by receiving amplified and processed digital EEG through wireless module. By contrast of traditional EEG detection system, the flexibility and portability of EEG detection system is added to this system. Compared to the existing portable EEG processing system, it is separated from upper compute and doesn't need living power supply. The whole system is more portable and smaller in size. Thus, this system possesses many characteristics such as: small volume, low power consumption, strong antigambling capacity and portability, etc.
\end{abstract}

Key words: OMAPL 138 processor, EEG; wireless module; portable; preprocessing

\section{Introduction}

EEG, a potential activity generated by nerve of brain containing substantial EEG activity information ${ }^{[1]}$, is the main basis for diagnosis of cerebral disease and also the main signal source of current study on brain-computer interface. Due to weak amplitude, EEG of human body is often submerged in various background noises ${ }^{[2]}$. Therefore, it should be obtained with special bioelectricity sensing and amplification collecting device. As far as traditional EEG detection device is concerned, it features large volume, high power consumption and cost. Further, wire mode is mostly used for data transmission ${ }^{[3-4]}$. Therefore, it is not convenient in obtaining EEG in real time. As a result, no matter for theoretical study or for monitoring patients in real time, study on portable EEG detection device is of great significance. Many research institutions have promoted wireless portable EEG detection system ${ }^{[5]}$. Speaking of portability of existing EEG acquisition and processing system, it is mainly represented by the fact that wireless module is used to receive and send data and signal mode recognition and feature extraction is implemented on lab view of personal computer with the consequence that it is not completely separated from upper computer. For this regard, OMAP-L138 portable EEG detection system is designed in this paper. With algorithm transplanted to the microprocessor, the upper computer is separated and the flexibility and practicability of EEG detection and processing system is increased. Besides, the display of signal, digital filtering and feature extraction is controlled through touch screen. The whole system features small volume, simple operation and portability. With real-time and dynamic row display of EEG, it is convenient in observation and studying.

\section{Overall System Scheme}

This system is mainly comprised of three parts, including EEG collection, wireless data transmission and processing. EEG signal collected by microelectrode is transmitted to simulation and amplifying filter circuit. DC polarization voltage could be easily generated at electrode edge. However, DC stability of circuit will be influenced by excessive polarization voltage. To eliminate DC polarization voltage generated by electrode edge, high-pass filter is introduced into amplifying circuit ${ }^{[6]}$. After amplifying EEG, amplifying circuit converts $\mathrm{A} / \mathrm{D}$ of signal, and firstly converts analog signal into digital one and then further processes it. Digital EEG is sent by MCU control wireless module (NRF24L01). Then, receiving module is controlled with microprocessor OMAPL138 to receive EEG data. The processing including FIR filtering and FFT of the EEG data will be implemented next. After the processed data is saved in SD card, the original and processed EEG could be displayed dynamically in real time through TFT color screen. For system flowchart, please refer to the Figure 1.

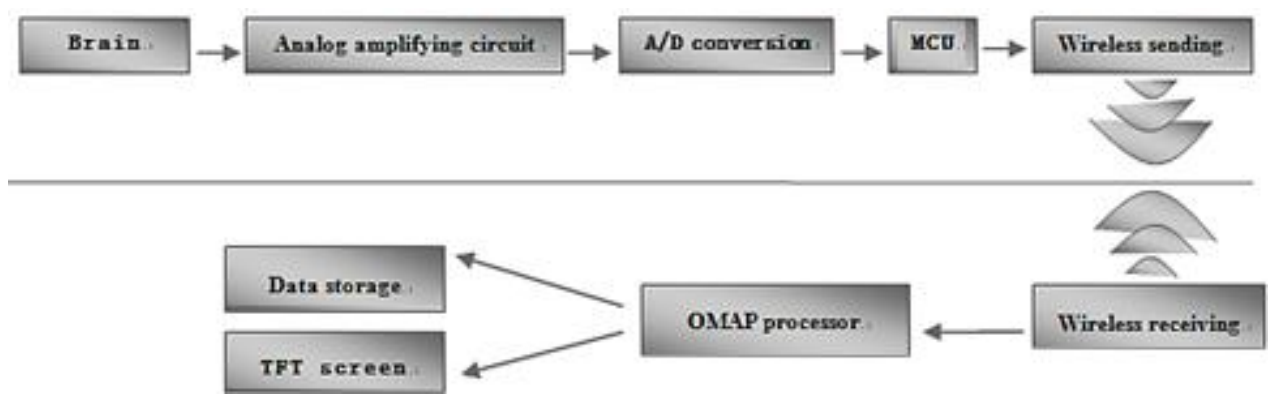

Figure 1: System Flowchart 


\section{System's Hardware Design}

\subsection{Front-end amplifying circuit}

Design is EEG amplifying circuit constituted by three stages of amplifications. The first stage refers to the preamplifier stage, preliminarily amplifying EEG by 100 times with differential amplifying circuit and coupling with the second level through RC high-pass filter circuit. High-pass filter is introduced to eliminate the useless signals outside of EEG frequency range, enhance system's antijamming capacity and avoid aliasing filtering. As for the second level of amplifying circuit, AD210 isolation and amplification stage circuit is adopted and the amplification factor is around 20 times. EEG enters into third-stage amplifying circuit through second-order voltage controlled voltage source low-pass filter. The third stage is amplifying circuit. Its amplification factor is adjustable and can be adjusted by around 20 times to satisfy the demand for AD conversion. Ultimately, it is accessed into AD conversion digital system through $50 \mathrm{~Hz}$ trapped wave circuit.

It is necessary to have high input impedance and CMRR at preamplifier stage. The higher the gain of preamplifier stage the smaller the influences on noises generated by latter circuits at all levels ${ }^{[7]}$. Therefore, it is necessary to increase the gain of prestage. However, the dynamic working range of amplifier restricts voltage gain of prestage, which is restricted by polarization voltage. Therefore, the prestage gain cannot be infinitely high. The new-type differential amplification circuit AD620 manufactured by Analog Devices shall be used as preamplifier stage while isolation amplifier AD210 manufactured by ADI is used as second-stage amplifier circuit. For principle diagram of circuits at all levels, please refer to the Figure 2.

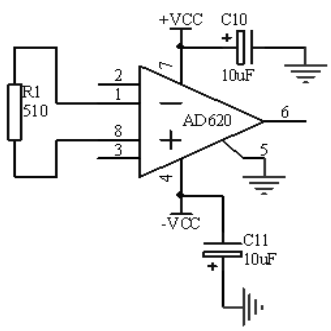

a) Prestage amplifying circuit

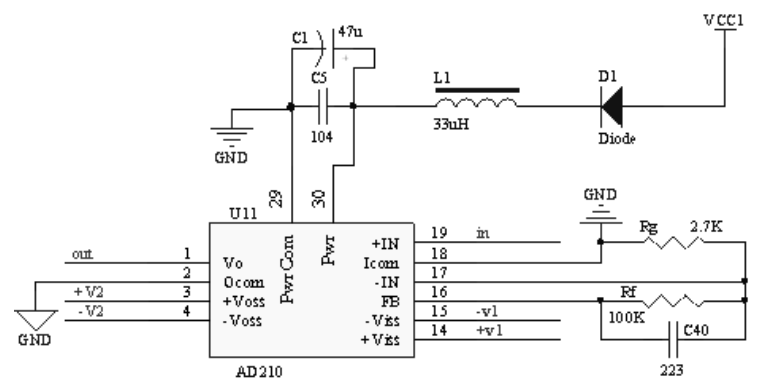

b) AD210 isolation amplifier stage circuit

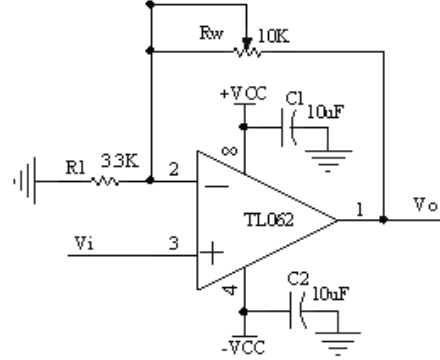

c) Third-stage adjustable amplifying circuit

Figure 2: Amplifying Circuit

\subsection{Wireless Module}

The portability of whole system is not only represented by useage and operation but also by volume. The smaller the system volume the more portable. Wireless module is introduced to EEG detection system, thus the whole detection system volume is smaller and portable. As a result, it is possible to realize portable performance. For the volume of nRF24L01 wireless module is small and its transmission speed is fast, its wireless communication speed can reach 2M (bps). Thus, it has higher transmission speed and lower current consumption than Bluetooth. Wireless module nRF24L01 is simple to operate. It is only necessary for the user to preset five GPIOs and one interrupt input pin for single-chip microcomputer, so that the function of wireless communication can be realized easily. Its characteristics could satisfy the requirements of system design. Therefore, wireless module nRF24L01 is used for this system to realize wireless data transmission. For its principle, please refer to the Figure 3.

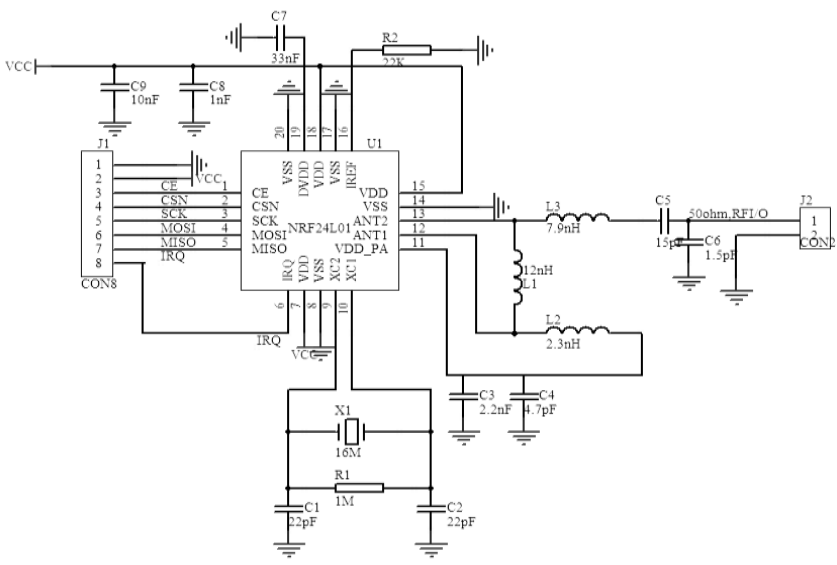

Figure 3:Principle Diagram of Wireless Module nRF24L01

\subsection{Data Storage}

In medical field, study EEG can help patients with diseases to let them pull through as far as quickly. Some brain diseases could be even treated by stimulating people's brain appropriately. Due to the specificity of EEG, the researcher cannot study EEG by directly obtaining it from the brain but should rely on EEG detection device. Therefore, this system saves the detected EEG while detecting it so as to analyze and process it. 


\section{International Journal of Science and Research (IJSR) \\ ISSN (Online): 2319-7064 \\ Index Copernicus Value (2013): 6.14 | Impact Factor (2014): 5.611}

SD card, regarded as portable mobile storage device, has already been widely applied into various media devices. The system's development board OMAPL138 integrates control access function of SD card. By compiling corresponding device drivers and application programs, SD card can be controlled and initialized. After EEG detection system is electrified and works stably, it processes the detected EEG and save it in SD card. By compiling different programs, SD card can realize more start-up and storage modes. For circuit diagram of SD card and system's development board OMAPL138 link, please refer to the Figure 4.

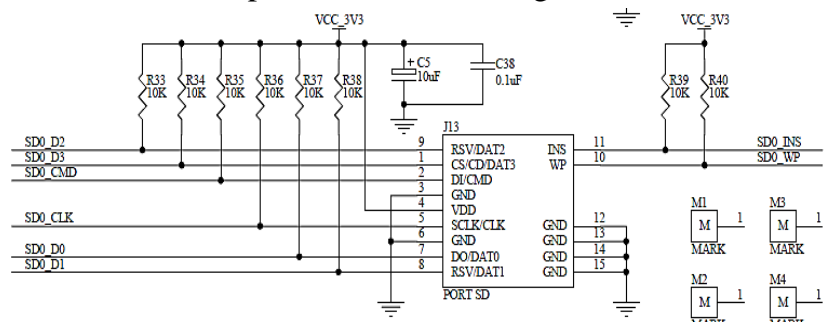

Figure 4: Circuit Diagram of SD Card Interface

\section{System Software Design}

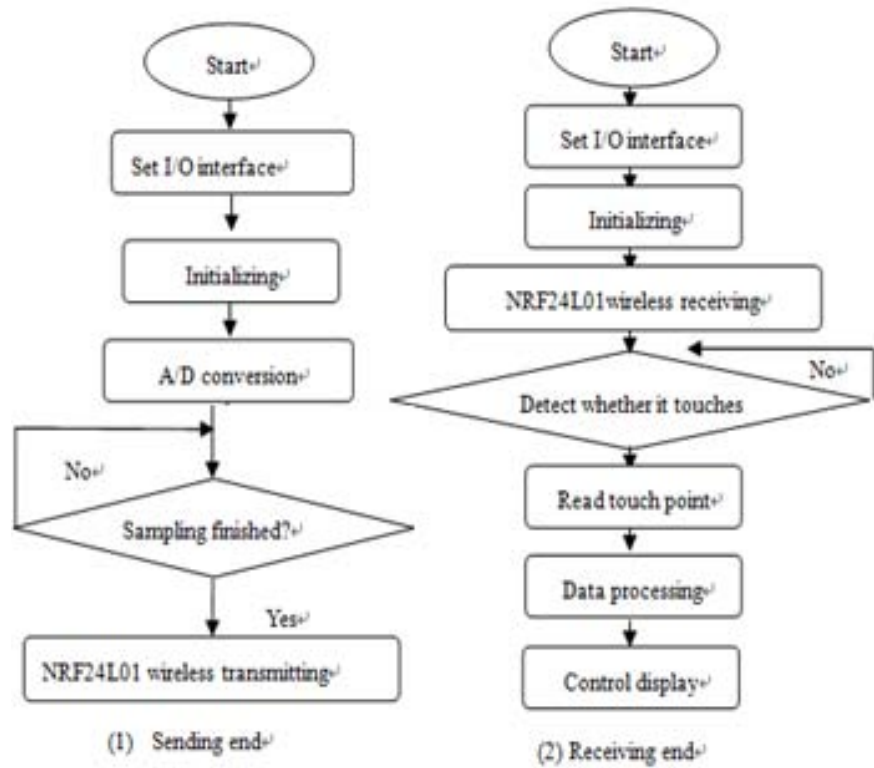

Figure 5:Program flow chart

Kernel chip OMAPL138 adopted for the system is a dicaryon embedded processor ${ }^{[8]}$. Whole system design includes DSP terminal platform building, building of ARM terminal embedded Linux software development, realization of communication between algorithm processing program design and dicaryon. Building of Linux operating system softwaredevelopment environment includes two parts: firstly, setting, cutting and transplanting of operating system; secondly, transplanting of bootstrap program, drive, kernel and root file system ${ }^{[9]}$. Considering the readability and transportability of program, C language is used as programming language to compile microprocessor application control program, FIR filtering, FFT algorithm program, drive program of wireless transmission module NRF24L01 and control program of TFT screen display and touch screen. IEEE802.15.4 standard communication protocol is adopted to compile the communication protocol satisfying the requirements of this design and corresponding hardware initializer. The key and difficult point is the transplanting ${ }^{[10]}$ of embedded Linux system kernel software and communication ${ }^{[11]}$ design between dicaryon. For specific software process, please refer to the Figure 5.

\section{System Experiment Result}

According to the mechanism of generation of EEG, it can be divided into two different types, namely evoked and spontaneous EEG, the former of which is stable and also more easily controlled than the latter one. Therefore, by combining the existing conditions and techniques of laboratory, stable VEP evoked by $16 \mathrm{~Hz}$ stimulation is used to test the designed system. According into international standard 10-20 lead EEG acquisition criterion, electrode of signal acquisition is placed at $\mathrm{O} 1$ area of subjects and two reference electrodes are pasted on left and right ear lobes of the subjects respectively. Then, primitive EEG enters into analog amplifying circuit through electrode, and then enters into analog-digital conversion module after amplified and processed, and then it is sent out with wireless module. After receiving the data, the kernel processor OMAPL138 begin processing and analyzing EEG data.

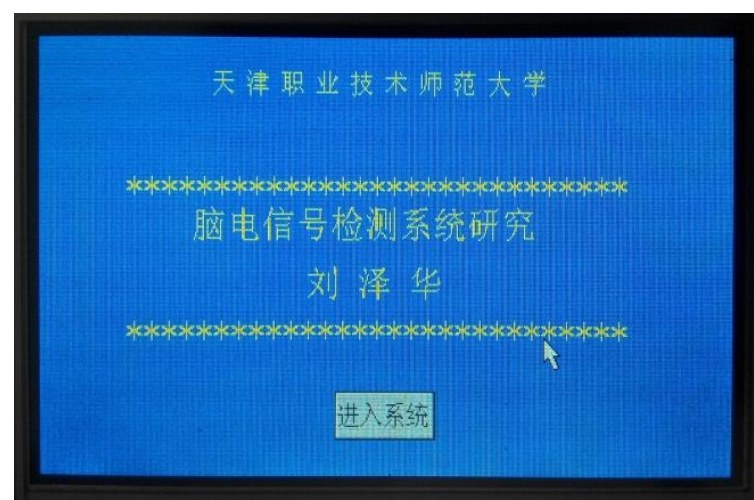

a) Initializing Interface

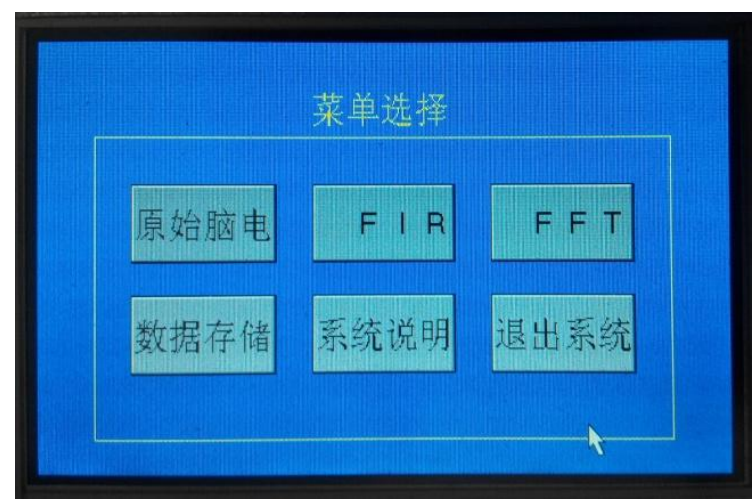

b) Main Interface

Figure 6:System Operation Interface

\subsection{FIR filtering processing experiment}

Through equal ripple approximation design method, 1 100-order FIR digital filter is designed for the system. The sampling frequency is 200. For experimental result, please refer to the Figure 7. 


\section{International Journal of Science and Research (IJSR) \\ ISSN (Online): 2319-7064 \\ Index Copernicus Value (2013): 6.14 | Impact Factor (2014): 5.611}

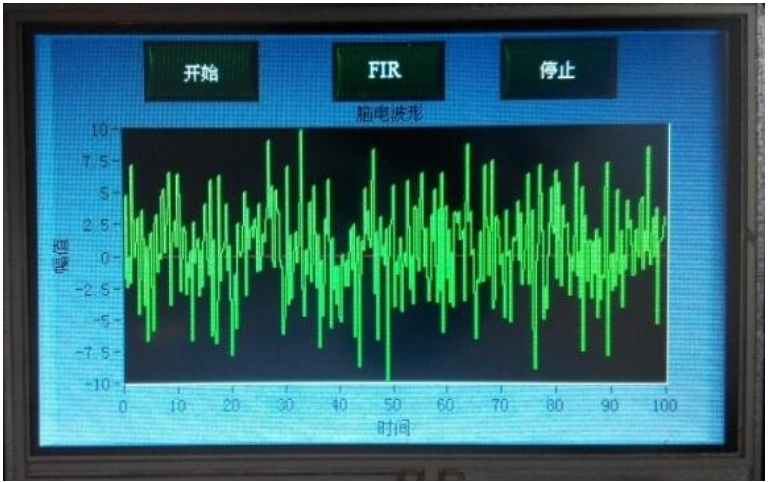

a) Primitive EEG

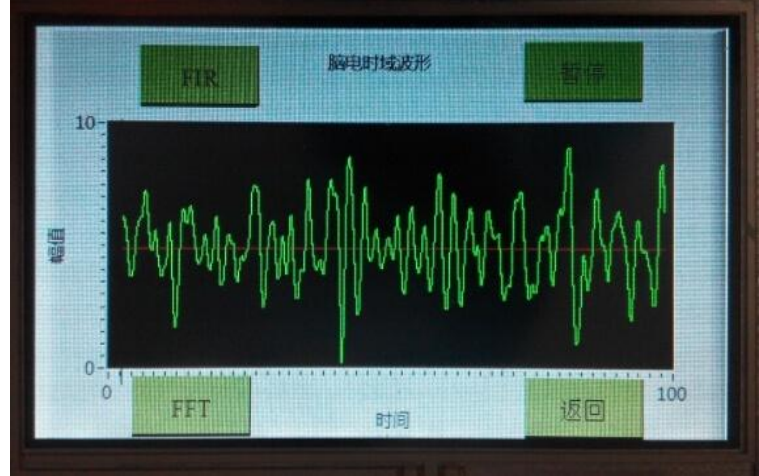

b) FIR Filtering

Figure 7: FIR Filtering of EEG

\subsection{FFT experiment}

Basic algorithm of FFT is divided into decimation-in-time and decimation-in-frequency. It is formed based on DFT. Through butterfly computation, its computation burden is greatly mitigated. FFT algorithm is used in the system is selected by time based -2 algorithm. For experimental result, please refer to the Figure 8.

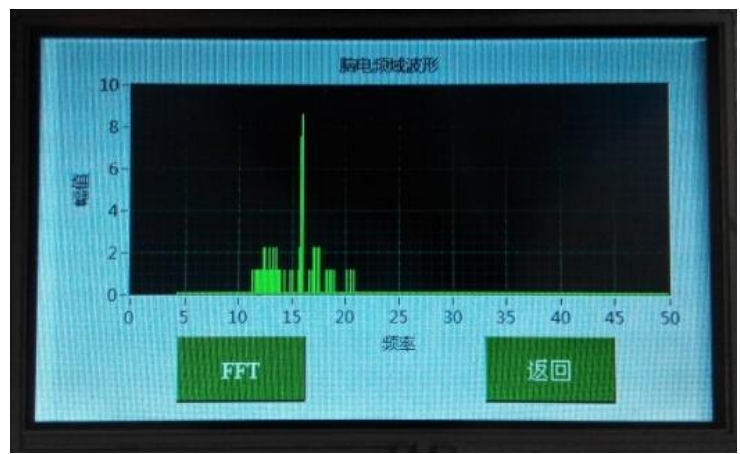

Figure 8: Frequency Domain of EEG

\section{Conclusions}

Based on the core OMAPL138, the wireless EEG detection system that features small volume, low power dissipation and real-time observation is designed for the system, thus the ideal effect is realized. Through touch screen control and switching, it is convenient in operation. This system possesses characteristics such as portability, low power dissipation and high integration density. It is applicable to occasions of medical diagnosis, helping the disabled and changing conditions. So, it is of high application value. Thus, the solid foundation for study on biological brain-computer interface in the future is laid.

\section{References}

[1] Xie Hong \& YangyangDong. EEG Acquisition and Transmission System Design Based on ADS1298 and Wi-Fi [J]. Modern Electronics Technique, 2013, 36(6):150-153.

[2] ZhenjiangLi\&JiminYang. Realization of STM32W108-based Wireless EEG Acquisition System $[J]$. Science and Technology Information, 2013(11):38-39.

[3] ShaobinWu, Gao Li \& Liu'anWang.EEG-based Study on Driving Fatigue Detection [J]. Journal of BI, 2009, 29(12):1072- 1075.

[4] Yin Gang, PingdongWu, JunqiangPeng, Et Al. Wearable Wireless EEG Monitor [J]. Microcomputer Information,2009, 25(4):276- 278.

[5] ZHANG L. Temporal Independent Component Analysis for Separating Noisy Signals [J]. Lecture Notes in Computer Science, 2004: 1064-1069.

[6] SUN S L,ZHANG C S. Adaptive Feature Extraction for EEG Signal Classification [J]. Medical \& Biological Engineering \& Computing, 2006, 44(10):931-935.

[7] Huang Qin, Zhou Ting, YiliangLiu. High CMRR EEG Amplifying Circuit Design [J]. Techniques of Automation and Applications, 2009, 28 (4): 77-79.

[8] Using the OMAP-Llx8 Boot loader Application Report. Texas Instruments, 2010.

[9] Zhu, Qing; Xu, Fei Yun. Research and Development of Portable Data Acquisition System Based on OMAP-LI38 [C].Mechanical Engineering and Technology. 2012.125: 185-192.

[10] TexasInstrumentsIne.OMAP-L137 Evaluation Module TeehniealReferenee.2010.

[11] Xinghong Li. OMAP-based Embedded System Development Data Acquisition and Processing [J].2008, 5 (7): 15-20.

\section{Author Profile}

\section{Ze-hua Liu}

Address: No. 1310, Dagu South Rd., Hexi District, Tianjin City Tianjin University of Technology and Education School of Automation

Postal Code: 300222 